\title{
LETTER Horizontal Partition for Scalable Control in Software-Defined Data Center Networks
}

\author{
Shaojun ZHANG ${ }^{\dagger a)}$, Julong LAN ${ }^{\dagger}$, Nonmembers, Chao $\mathrm{QI}^{\dagger}$, Student Member, and Penghao $\mathrm{SUN}^{\dagger}$, Nonmember
}

\begin{abstract}
SUMMARY Distributed control plane architecture has been employed in software-defined data center networks to improve the scalability of control plane. However, since the flow space is partitioned by assigning switches to different controllers, the network topology is also partitioned and the rule setup process has to invoke multiple controllers. Besides, the control load balancing based on switch migration is heavyweight. In this paper, we propose a lightweight load partition method which decouples the flow space from the network topology. The flow space is partitioned with hosts rather than switches as carriers, which supports fine-grained and lightweight load balancing. Moreover, the switches are no longer needed to be assigned to different controllers and we keep all of them controlled by each controller, thus each flow request can be processed by exactly one controller in a centralized style. Evaluations show that our scheme reduces rule setup costs and achieves lightweight load balancing.

key words: software-defined networking, control plane, scalability, load partition, load balancing
\end{abstract}

\section{Introduction}

Software-defined networking (SDN) [1] is becoming prevalent in data center networks due to its support of agile innovation and flexible management. However, the control plane of SDN suffers from scalability problems [2]. In data centers where the size of the networking area grows slowly, the leading issue of scalability is controller overload due to the explosion of new flows in the network, which results in excessively long response time for flow setup requests.

To address such issue, numerous distributed control planes with a cluster of controllers have been introduced [3], [4]. They partition the network into several domains by assigning switches to different controllers, with each controller only responsible for the flow setup requests pertaining to the switches in its domain. In this way the control load is distributed to multiple controllers and the response delay decreases. Besides, to adapt to the traffic fluctuation in the network, dynamic switch assignment based on switch migration [5] has been proposed to balance the load among controllers for lower delay [6], [7].

However, there are still some challenges to the switches partition mechanism and the corresponding load balancing scheme. (i) Since the network is split into several control domains, flows transmitting across the network may

\section{Manuscript received December 18, 2017.}

Manuscript revised February 4, 2018.

Manuscript publicized March 7, 2018.

${ }^{\dagger}$ The authors are with National Digital Switching System Engineering and Technological R\&D Center, Zhengzhou, 450002 China.

a) E-mail: zhangsj07@126.com

DOI: 10.1587/transinf.2017EDL8273 run through multiple domains and the rule setup process involves all the controllers along the flow path. Such procedure complicates the rule setup process and consumes extremely high control resources. (ii) The control load is partitioned in a coarse-grained style where the granularity of load is the overall flow requests generated at a switch. In this way, the migration of load among controllers has to be conducted in a heavy and coarse-grained manner, which forces too many unnecessary flow requests into the migration process.

In this letter, we demonstrate a graceful control load partition scheme called horizontal partition (HP). HP decouples control load from network topology, so that the load can be partitioned flexibly without the assignment of switches. Evaluations show that HP can reduce the rule setup costs and can achieve lightweight load balancing.

\section{Motivation}

The SDN framework can be regarded as a service system whose service provider is the control plane. Although the load of the control plane comes from the flow setup requests generated at switches, the real serving objects of the control plane are the flows produced by users. The network of switches are tools to implement these services, i.e., forwarding the packets of each flow through the switches. The flow setup requests are also triggered by the arrival of new flows. Therefore, the load of the control plane actually corresponds to the flow space of the network.

To deal with scalability problems, distributed control plane is deployed and the flow space is partitioned and allocated to different controllers. Currently, the flow space is partitioned by using switches as carriers and distributing switches to controllers, which can be called vertical partition (VP, Fig. 1, left). VP is a coarse-grained method and what is worse, the network topology is also partitioned and each controller can only controls a subset of the network, which leads to the control stretching problem. For example, for the flow from $\mathrm{h} 1$ to $\mathrm{h} 2$, the path in the network is $\mathrm{s} 1 \rightarrow \mathrm{s} 2 \rightarrow \mathrm{s} 3$. Since $\mathrm{s} 1$ and $\mathrm{s} 2$ belong to $\mathrm{c} 1$ while $\mathrm{s} 3$ belongs to $\mathrm{c} 2, \mathrm{c} 1$ and c2 are both needed to complete this flow setup service. In general, all the controllers that the switches along the path belong to will be involved in the flow setup process, which complicates the rule setup process, consumes high control resources and introduces long response delay.

Unlike VP, HP (Fig. 1, right) decouples flow space from the network topology and keeps all the switches con- 


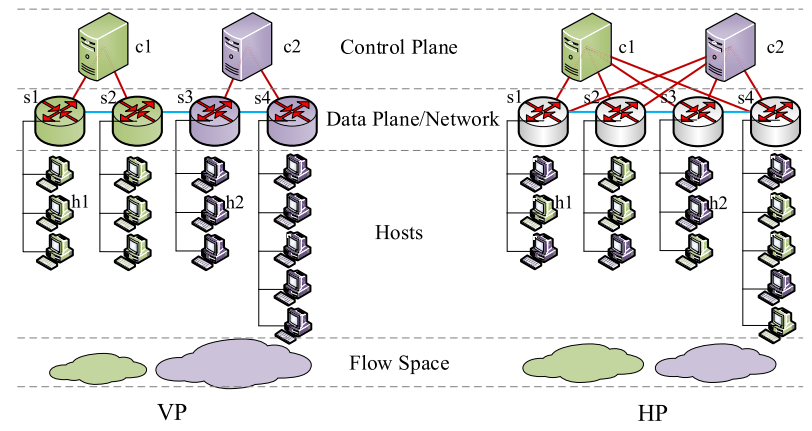

Fig. 1 Comparison between VP and HP.

trolled by each controller. In this way, HP takes only one controller to complete the service for any flow in a centralized control logic, which complies with the original intent of SDN. For the same flow from h1 to h2 with the path $\mathrm{s} 1 \rightarrow \mathrm{s} 2 \rightarrow \mathrm{s} 3$, $\mathrm{c} 1$ is enough to accomplish this control task.

Moreover, without the binding of switches, HP can partition the flow space in a more flexible way. As for the control load balancing problem, HP chooses hosts as the carriers of the flows to partition the flow space, which is fine-grained and can keep the whole network behavior of one user under a single controller. Besides, the flows from certain host can be distinguished by the "IN_PORT" field in the flow setup requests, which can simplify the load distributing procedure.

\section{HP Framework}

We introduce HP-proxy to the current SDN framework (Fig. 2), which sits between the data plane and the control plane and can distribute the flow setup requests generated in switches to different controllers. To remain transparent to switches whose structure can hardly change, HP-proxy acts as a controller to the underlying data plane. It receives flow setup request (e.g. Packet-In) from the switches, sends it to the corresponding controller, and responds with the rule (e.g. Flow-Mod) generated on this controller.

As stated before, HP uses hosts as the carriers of the flows to partition the flow space. We define a slice as the flow space associated to a host. All the flows generated at the host belong to its corresponding slice ${ }^{\dagger}$. Then a slice can simply be identified with the parameter (Switch, Port) that its associated host connects to.

The slice table is a database to store the load allocation policy $\{($ Switch, Port $):$ Controller $\}$, i.e. which controller should a slice be allocated to. Upon the arriving of a new flow setup request, HP-proxy matches the (Switch, Port) parameter of the flow against the slice table, and sends it to the right controller. The load allocation policy is calculated by the control load balancing module according to the load of each controller and the size of each slice. HP-proxy updates the policy dynamically to adapt to the flow fluctuation of the

${ }^{\dagger}$ For flows generated in network, e.g. LLDP related flows, since they are usually slight, we can process them at HP-proxy and publish the related network state information to the control plane.

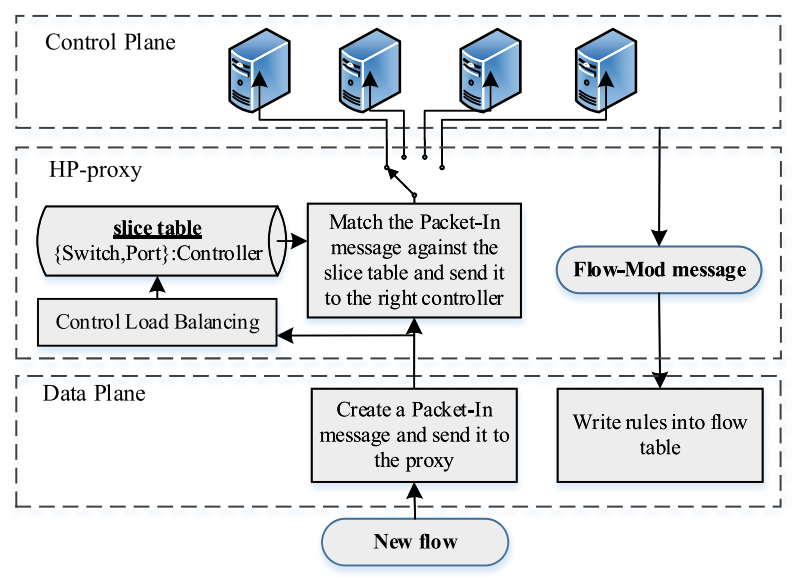

Fig. 2 HP framework

hosts and keep the load across the control plane balanced.

\section{Control Load Balancing}

The objective of control load balancing is to properly adjust the assignment of slices to controllers such that the average response time is minimized.

The SDN network is modelled by $M$ controllers $\left\{c_{1}, c_{2}, \ldots, c_{M}\right\}, K$ switches and $N$ hosts $\left\{h_{1}, h_{2}, \ldots, h_{N}\right\}$. All the controllers are assumed to have equal processing capacity $c$, i.e. the number of requests that a controller can handle per time unit.

The request demand of host $h_{j}$ is assumed to follow a Poisson process with $\lambda_{j}$, which is equal to the size of its slice $s_{j}$. We employ a binary matrix $X$ to denote the assignment between slices and controllers, where $x_{i j}=1$ if $s_{j}$ is assigned to $c_{i}$ and $x_{i j}=0$ otherwise. Then the load of $c_{i}$ can be represented as:

$$
l_{i}=\sum_{j=1}^{N} \lambda_{j} x_{i j}
$$

Because of the high bisection bandwidth in data center networks, the propagation delay is usually negligible compared with the controller processing time [7], therefore we employ only the latter to model the response time. We assume that a controller can be modelled as an $\mathrm{M} / \mathrm{M} / 1$ queue, then the average response time of the control plane can be calculated as:

$$
\bar{\tau}=\frac{\sum_{i=1}^{M} \frac{l_{i}}{c-l_{i}}}{\sum_{j=1}^{N} \lambda_{j}}
$$

The control load balancing problem can be defined as:

$$
\begin{array}{ll}
\min & \bar{\tau} \\
\text { s.t. } & l_{i} \leq c, \forall i
\end{array}
$$




$$
\begin{aligned}
& \sum_{i=1}^{M} x_{i j}=1, \forall j \\
& x_{i j} \in\{0,1\}, \forall i, j
\end{aligned}
$$

Constraint (4) ensures that no controller will be overloaded. Constraint (5) restricts that each slice can be assigned to only one controller.

\section{Numerical Results}

We now conduct a numerical analysis of the HP scheme. We simulate a network with 5 controllers and 36 switches. The simulator is implemented in Python and the topology is a randomly generated Barabasi-Albert network using the NetworkX library [8]. Each switch connects with 10 hosts. Each host generates flows randomly with the mean of 100 and sends them to other hosts at random. The controller capacity is set to 10000 and each flow selects the shortest path.

The optimal assignment of slices to controllers is calculated by utilizing the state-of-the-art optimizer Gurobi [9]. We conduct the experiment for 50 times both in HP and VP (by replacing slices with switches) to compare their performance.

Figure 3 illustrates the average number of involved controllers to respond to a flow setup request. We can see that VP needs 2.8 controllers on average to respond to one flow setup request, which means high resource consumption. On the other hand, without the partition of the network topology, HP can complete each response with only one controller. Figure 4 shows the average response time of the control plane in HP and VP. Although the request rates

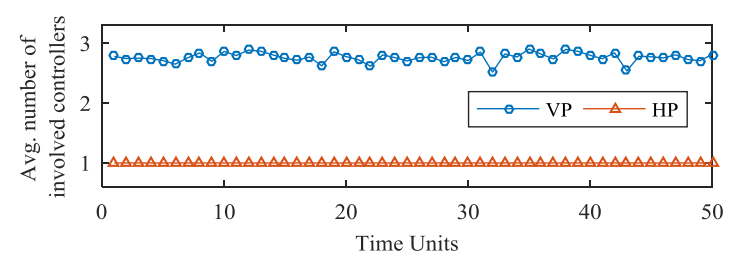

Fig. 3 Average number of involved controllers for a flow setup request

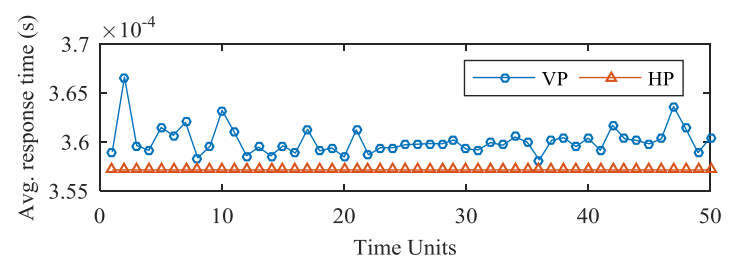

Fig. 4 Average response time after load balancing

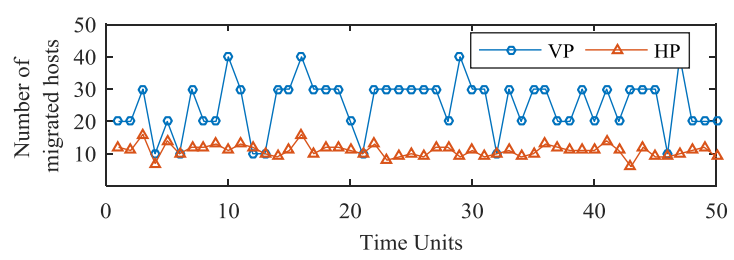

Fig. 5 Number of migrated hosts during load balancing of the hosts fluctuate dynamically, the load among the controllers has been balanced through reassignment of switches (in VP) or slices (in HP), thus the response time of VP and HP both experiences low fluctuation. However, due to the fine-grained load partition, the load balancing performance of HP is more remarkable than that of VP. Figure 5 shows the total number of migrated hosts during the load balancing. The switch migration method in VP is coarse-grained and forces too many hosts, while slice migration in HP can achieve load balancing in a fine-grained and lightweight manner.

\section{Conclusion}

This letter gives a first attempt to decouple flow space from the data plane. The proposed flow space partition mechanism can achieve effective control and lightweight load balancing, which improves the scalability of the control plane. In the future, we will focus on other dimensions of flow space partition to support a more flexible and scalable control plane.

\section{Acknowledgments}

This work was supported by the Foundation for Innovative Research Groups of the National Natural Science Foundation of China (No. 61521003) and the National HighTech Research \& Development Program of China (No. 2015AA016102).

\section{References}

[1] D. Kreutz, F.M.V. Ramos, P.E. Verissimo, C.E. Rothenberg, S. Azodolmolky, and S. Uhlig, "Software-defined networking: a comprehensive survey," Proc. IEEE, vol.103, no.1, pp.14-76, 2015. DOI: 10.1109/JPROC.2014.2371999

[2] M. Karakus and A. Durresi, "A survey: Control plane scalability issues and approaches in Software-Defined Networking (SDN)," Computer Networks, vol.112, pp.279-293, 2017. DOI: 10.1016/j.comnet. 2016.11.017

[3] A.S.W. Tam, K. Xi, and H.J. Chao, "Use of devolved controllers in data center networks," Proc. 30th INFOCOM WKSHPS, Shanghai, China, pp.596-601, 2011. DOI: 10.1109/INFCOMW.2011.5928883

[4] P. Berde, W. Snow, G. Parulkar, M. Gerola, J. Hart, Y. Higuchi, M. Kobayashi, T. Koide, B. Lantz, B. O'Connor, and P. Radoslavov, "ONOS: towards an open, distributed SDN OS," Proc. 3rd HotSDN, New York, NY, USA, pp.1-6, 2014. DOI: 10.1145/2620728.2620744

[5] A. Dixit, F. Hao, S. Mukherjee, T.V. Lakshman, and R. Kompella, "Towards an elastic distributed SDN controller," ACM SIGCOMM Computer Communication Review, vol.43, no.4, pp.7-12, 2013. DOI: $10.1145 / 2534169.2491193$

[6] X. Gao, L. Kong, and W. Li, "Traffic load balancing schemes for devolved controllers in mega data centers," IEEE Trans. Parallel Distrib. Syst., vol.28, no.2, pp.572-585, 2017. DOI: 10.1109/TPDS.2016. 2579622

[7] T. Wang, F. Liu, and H. Xu, "An efficient online algorithm for dynamic SDN controller assignment in data center networks," IEEE/ACM Trans. Netw., vol.25, no.5, pp.2788-2801, 2017. DOI: 10.1109/ TNET.2017.2711641

[8] http://networkx.github.io

[9] http://www.gurobi.com 